\title{
Risk factors for non-reaching of ileal pouch to the anus in laparoscopic restorative proctocolectomy with handsewn anastomosis for ulcerative colitis
}

\author{
Shigenobu Emoto, Keisuke Hata, Hiroaki Nozawa, Kazushige Kawai, Toshiaki Tanaka, Takeshi Nishikawa, \\ Yasutaka Shuno, Kazuhito Sasaki, Manabu Kaneko, Koji Murono, Yuuki Iida, Hiroaki Ishii, Yuichiro Yokoyama, \\ Hiroyuki Anzai, Hirofumi Sonoda, Soichiro Ishihara \\ Department of Surgical Oncology, The University of Tokyo, Tokyo, Japan
}

Background/Aims: Restorative proctocolectomy (RPC) with ileal pouch-anal anastomosis and handsewn anastomosis for ulcerative colitis requires pulling down of the ileal pouch into the pelvis, which can be technically challenging. We examined risk factors for the pouch not reaching the anus. Methods: Clinical records of 62 consecutive patients who were scheduled to undergo RPC with handsewn anastomosis at the University of Tokyo Hospital during 1989-2019 were reviewed. Risk factors for non-reaching were analyzed in patients in whom hand sewing was abandoned for stapled anastomosis because of nonreaching. Risk factors for non-reaching in laparoscopic RPC were separately analyzed. Anatomical indicators obtained from presurgical computed tomography (CT) were also evaluated. Results: Thirty-seven of 62 cases underwent laparoscopic procedures. In 6 cases (9.7\%), handsewn anastomosis was changed to stapled anastomosis because of non-reaching. Male sex and a laparoscopic approach were independent risk factors of non-reaching. Distance between the terminal of the superior mesenteric artery (SMA) ileal branch and the anus $>11 \mathrm{~cm}$ was a risk factor for non-reaching. Conclusions: Laparoscopic RPC with handsewn anastomosis may limit extension and induction of the ileal pouch into the anus. Preoperative CT measurement from the terminal SMA to the anus may be useful for predicting non-reaching. (Intest Res 2022;20:313-320)

Key Words: Handsewn anastomosis; Laparoscopy; Restorative proctocolectomy; Ulcerative colitis

\section{INTRODUCTION}

Restorative proctocolectomy (RPC) with ileal pouch-anal anastomosis (IPAA) is a procedure used to treat patients with ulcerative colitis with refractory disease or when the patients develop neoplasms. This procedure was first described by Parks and Nicholls in 1978; the whole diseased colon and rectum are removed, while maintaining intestinal continuity without a permanent stoma. There are 2 techniques by which

Received December 11, 2020. Revised January 5, 2021.

Accepted January 12, 2021.

Correspondence to Shigenobu Emoto, Department of Surgical Oncology,

The University of Tokyo, 7-3-1, Hongo, Bunkyo-ku, Tokyo 113-8655, Japan.

Tel: +81-3-3815-5411, Fax: +81-3-3811-6822, E-mail: emotos-sur@h.u-

tokyo.ac.jp
IPAA can be performed. As described by Parks and Nicholls, the procedure involves a mucosectomy with handsewn anastomosis, sometimes called ileal pouch-anal anastomosis (IAA). The second technique, which is called ileal pouch-anal canal anastomosis (IACA), uses a double-stapled anastomosis and was developed to improve postoperative function by preserving the anal transition zone and mucosa of the rectal stump; in this technique, a stapled anastomosis is made without mucosal resection. ${ }^{2}$ Previous reports designated the history of colorectal neoplasia as a risk factor associated with developing other neoplasia in the residual rectum or anastomosis after radical colectomy. Therefore, handsewn anastomosis with mucosectomy is considered desirable for cancer cases.

In handsewn anastomosis, the ileal pouch must be mobi- 
lized such that it may be extended by at least $2-4 \mathrm{~cm}$ into the pelvis, which may be challenging to achieve in all cases due to an inadequate length of the pouch pedicle. As a solution, a stapled anastomosis can be performed, which involves the omission of mucosectomy and improves the feasibility of anastomosis. A stapled anastomosis ensures that less tension is induced than with a handsewn anastomosis. ${ }^{4}$ Therefore, in some cases, handsewn anastomosis with mucosectomy must be aborted and switched to stapled anastomosis or the scheduled procedure changed to abdominoperineal resection with a permanent ileostomy. ${ }^{5}$

Several methods for enabling the ileal pouch to reach the anus have been reported, including sufficient mobilization of the small intestine, division of the mesenteric vessels supplying blood to the pouch, and performing transmesenteric incisions. ${ }^{6-16}$ Cadaveric studies demonstrated the efficacy and safety of division of the ileocolic artery (ICA) or branches of the superior mesenteric artery (SMA), ${ }^{6,710,12,16}$ and surgical results of each technique in single-center studies have been reported. $5,8,9,11,13,14,17$

Only few studies have investigated the risk of required abandonment of handsewn anastomosis because of the inability of the ileal pouch to reach the anus. Older age and severe obesity are established risk factors. ${ }^{5}$ Ohira et al. ${ }^{17}$ reported that the distance between the terminal branch of the ICA and the anal verge (AV), measured using axial computed tomography (CT), was a useful predictor of the difficulty to extend the ileal pouch to the anus.

More recently, the wider implementation of laparoscopic colorectal surgery has enabled laparoscopic IPAA for ulcerative colitis. ${ }^{18-21}$ It remains unclear whether the laparoscopic approach itself is a risk factor for the pouch not reaching the anus, as well as what other risk factors are associated with the inability of the pouch to reach the anus in laparoscopic surgery. Therefore, in this study, we aimed to examine whether laparoscopic RPC with IPAA was a risk factor for the inability of the pouch to reach the anus and sought to identify other risk factors associated with this phenomenon.

\section{METHODS}

\section{Surgical Procedures}

In our hospital, we perform elective total proctocolectomy with mucosectomy and handsewn IPAA as the first-line surgery for patients who develop colitis-associated cancer or dysplasia. For refractory inflammation or acute exacerbation, most patients first undergo subtotal colectomy as an emergency operation, and remaining proctocolectomy with double-stapling anastomosis without mucosectomy as a secondary surgery 3-6 months later. However, some patients with severe inflammation around the lower rectum undergo handsewn anastomosis with mucosectomy. In this procedure, we make a 15$\mathrm{cm}$ long ileal J-pouch, preserving the ICA. ${ }^{22}$ The apex of the pouch is shifted 2 finger-breadths below the inferior border of the pubic symphysis. The mesentery of the small intestine is fully mobilized. If the pouch is unable to reach this far, 1-3 SMA branches to the ileal pouch are divided and the mesentery is fenestrated, while taking maximal care not to sacrifice blood perfusion of the intestine. ${ }^{8}$ Patients with neoplasia or severe inflammation around lower rectum should not be treated with stapled IPAA in which rectal mucosa cannot be removed completely. Therefore, in these patients, our first alternative procedure in case the pouch does not reach the anus is open conversion in which we can keep pushing the J-pouch into the pelvis safely by hand until transanal anastomosis is completed. If the pouch does not reach even after open conversion, the surgical form should be changed to abdominoperineal resection with a permanent ileostomy is needed.

On the other hand, in patients without neoplasia or severe inflammation around lower rectum, we convert scheduled handsewn IPAA to stapled IPAA before trying handsewn anastomosis or open conversion.

\section{Methods to Determine Risk Factors for Non- Reaching of the J-Pouch in Handsewn Anastomosis}

The clinical records of 62 consecutive patients who were scheduled to undergo RPC with handsewn anastomosis for ulcerative colitis at the University of Tokyo Hospital between 1989 and 2019 were retrospectively reviewed.

The risk factors for non-reaching were assessed by univariate and multivariate analyses for patients in whom the procedure had to be changed from handsewn anastomosis to stapled anastomosis because the pouch did not reach the anus. Age, sex, height, body mass index, surgical approach, and operative indications were evaluated. The risk factors for nonreaching of the ileal pouch to the anus in laparoscopic proctocolectomy with handsewn anastomosis were separately analyzed.

To investigate anatomical factors, we measured the distance between the horizontal contrast-enhanced CT slices that contain the root of the SMA (rSMA) and the terminal of the ileal branch of the SMA (tSMA; identifiable on the most caudal slice 
in the arterial phase of contrast-enhanced CT scans), and the upper margin of the anal canal (AC) (Fig. 1). The distance between the ischium at the level of the femoral head (d-Sci) in the axial view (Fig. 2A), from the promontory angle to the suprapubic margin (d-inlet), and from the coccyx to the inferior pubic margin (d-outlet) in the sagittal view, were also mea-

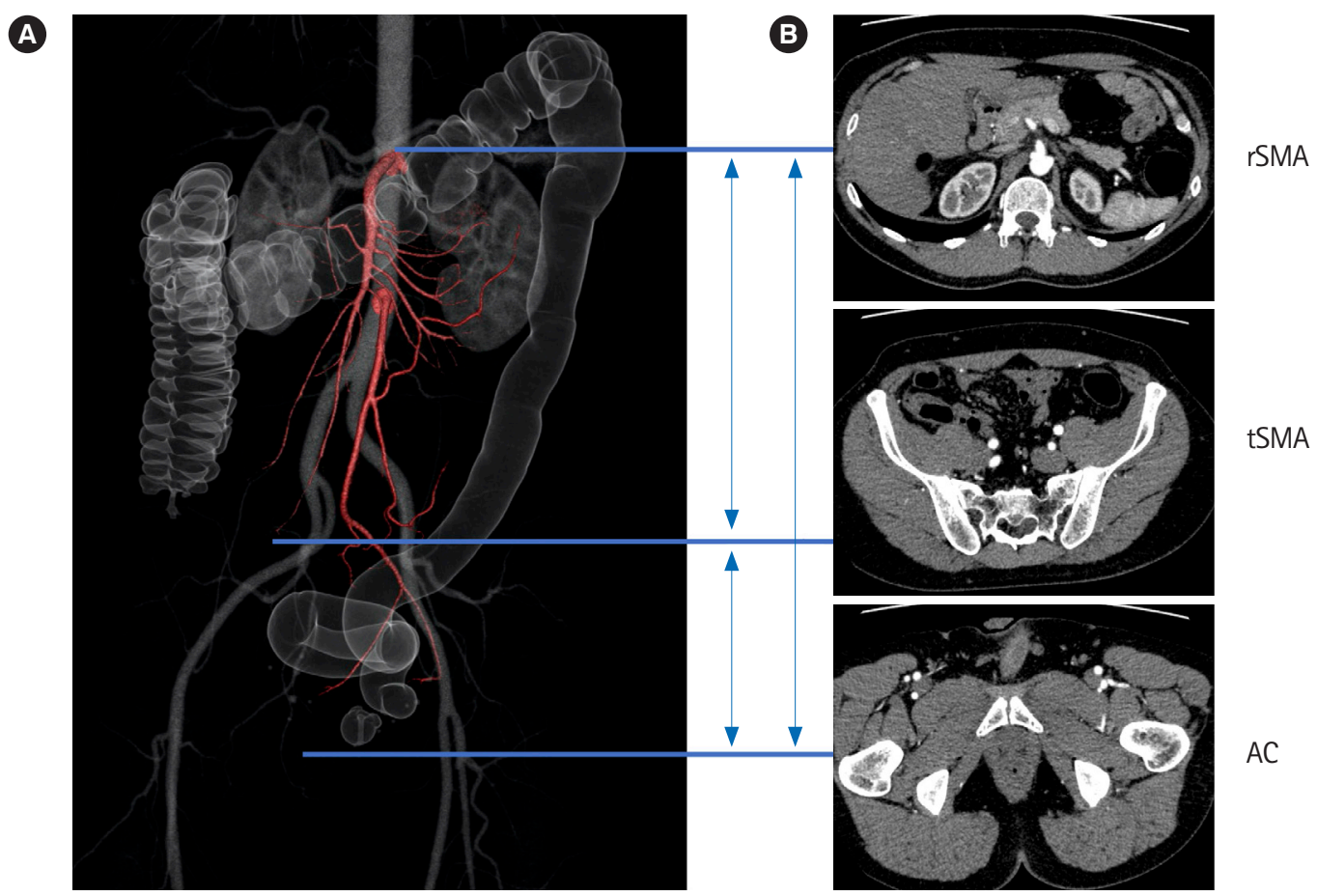

Fig. 1. The distances between the horizontal contrast-enhanced computed tomography (CT) slices that contain the root of the SMA (rSMA) and the terminal of the ileal branch of the SMA (tSMA), which can be identified on the most caudal slice in the arterial phase of contrastenhanced CT scans, and the upper margin of the anal canal (AC) were measured. SMA, superior mesenteric artery.

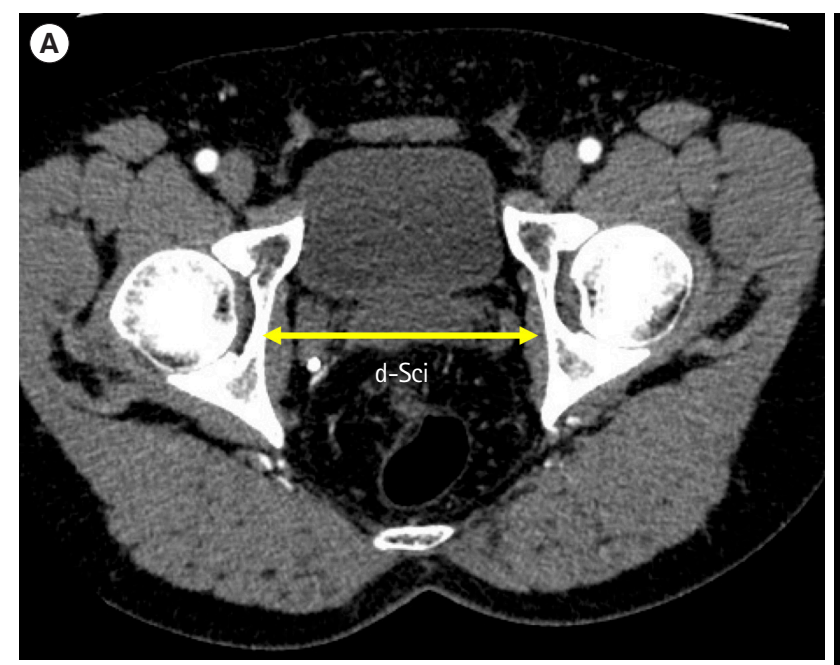

Fig. 2. The distances between the ischium at the level of the femoral head ( $d$-Sci) in the axial view (A), from the promontory angle to the suprapubic margin (d-inlet), and from the coccyx to the inferior pubic margin (d-outlet), in the sagittal view (B), were also measured.

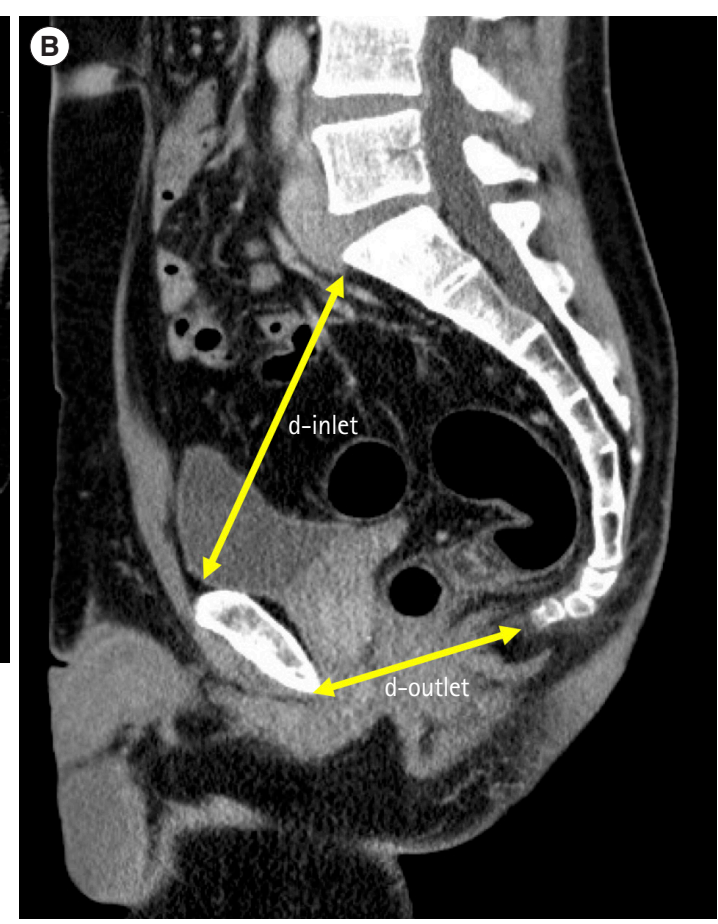


sured (Fig. 2B). CT studies taken after 2012 were performed using a multi-detector row (4-320 rows) helical CT scanner with a tube voltage of $120 \mathrm{kVp}$ and a slice thickness of $5 \mathrm{~mm}$. The contrast-enhanced images were reconstructed with a slice thickness of $1.25 \mathrm{~mm}$. Three-dimensional reconstruction of SMA arterial branch was routinely performed. Before 2011, a slice thickness was $5 \mathrm{~mm}$ or $10 \mathrm{~mm}$. Radiological assessment was performed by 2 board-certified colorectal surgeons (S.E. and K.M.; both of them were 16 years of experience).

This study was approved by the local ethics committee of the University of Tokyo Hospital (approval No. 3252-(9)) and written informed consent for data use was obtained from all the patients enrolled in the study. This study was conducted according to the principles of the Declaration of Helsinki.

\section{Statistical Analyses}

Relationships between clinicopathological features and nonreaching of the J-pouch to the anus were evaluated using the chi-square test and Fisher exact test. Multivariate analyses were performed using logistic regression modelling. The $t$-test was used to compare continuous variables. $P$-values $<0.05$ were considered statistically significant. All statistical analyses were performed using the JMP program, version 14.0 (SAS Institute, Cary, NC, USA). Continuous values are expressed as mean \pm standard deviation.

\section{RESULTS}

\section{Patient Characteristics}

Table 1 presents the baseline characteristics of the patients.

Table 1. Patient Characteristics

\begin{tabular}{lc}
\hline Characteristic & \multicolumn{1}{c}{ Value } \\
\hline No. of patients & 62 \\
Age $(\mathrm{yr})$ & $48(20-77)$ \\
Male sex & $38(61.3)$ \\
Body mass index $\left(\mathrm{kg} / \mathrm{m}^{2}\right)$ & $21(14-32)$ \\
Operative indication: cancer/dysplasia & $49(79.0)$ \\
Surgical approach: laparoscopy & $37(59.7)$ \\
Operative duration (min) & $489(195-1,065)$ \\
Bleeding (g) & $400(20-2,200)$ \\
Division of mesenteric vessels of pouch & $39(62.9)$ \\
Conversion to stapled anastomosis & $6(9.7)$ \\
Covering stoma & $54(87.1)$ \\
\hline
\end{tabular}

Values are presented as median (range) or number (\%).
Thirty-seven of 62 patients underwent laparoscopic surgery, which included 4 hand-assisted laparoscopic surgeries and 1 robot-assisted laparoscopic surgery. Laparoscopic J-pouch surgery was first performed in 2003 in our department, and since 2012, all 31 patients have undergone laparoscopic surgery.

In 39 patients (62.9\%), the SMA branch was dissected and the mesentery was fenestrated to allow extension of ileal pouch up to the anus. In 6 cases (9.7\%), the scheduled handsewn anastomosis was changed to stapled anastomosis because we assessed the ileal pouch could not reach the anus, even after dissection of the SMA branch. In these 6 patients, even mucosectomy or open conversion was attempted because no neoplasia nor severe inflammation was existed in lower rectum. We decided to convert to stapled IPAA following our strategy described in the methods section. No patients underwent abdominoperineal resection or open conversion due to non-reaching.

\section{Risk Factors for Non-Reaching of the J-Pouch in Handsewn Anastomosis}

Risk factors for non-reaching of the J-pouch to the anus were analyzed (Table 2). In univariate analysis, male sex $(P=0.01)$ and laparoscopic surgery $(P=0.01)$ were risk factors of nonreaching. In multivariate analysis, male sex and laparoscopic surgery were independent risk factors of non-reaching.

In terms of surgical outcome, anastomotic leakage, anastomotic stenosis, small bowel obstruction, and pouchitis occurred in $1,3,15$, and 9 patients, respectively. One of the 6 patients in whom handsewn anastomosis was abandoned due to nonreaching developed cancer in the residual rectal mucosa at 3 years after IPAA, and he underwent abdominoperineal resection as a salvage surgery.

\section{Anatomical Features of Patients with Non-Reaching in Laparoscopic Handsewn Anastomosis}

Anatomical features of the 37 patients who underwent laparoscopic surgery were also analyzed. The tSMA-to-AC and the rSMA-to-AC distances $(\mathrm{mm})$ were $98.5 \pm 30.4,305.0 \pm 19.3$, and those of d-Sci, d-inlet, and d-outlet were $108.0 \pm 11.0,114.0 \pm 11.8$, and $86.6 \pm 7.8$, respectively. When these were analyzed as continuous variables, no significant differences between reaching and non-reaching patients were detected. However, in cases in whom the pouch did not reach, the tSMA-AC tended to be long and the d-outlet tended to be short (Table 3).

Patients who underwent laparoscopic surgery were separately analyzed to assess the non-reaching risk (Table 4). None of the patients required conversion to open laparotomy. Divi- 
Table 2. Risk Factors for Conversion from Handsewn to Stapled Anastomosis

\begin{tabular}{|c|c|c|c|c|c|c|}
\hline \multirow[b]{2}{*}{ Risk factor } & \multirow[b]{2}{*}{ No. } & \multicolumn{2}{|c|}{ Univariate analysis } & \multicolumn{3}{|c|}{ Multivariate analysis } \\
\hline & & $\begin{array}{l}\text { Conversion to stapled } \\
\text { anastomosis, No. (\%) }\end{array}$ & $P$-value & OR & $95 \% \mathrm{Cl}$ & $P$-value \\
\hline Total & 62 & $6(9.7)$ & & & & \\
\hline \multicolumn{7}{|l|}{ Age (yr) } \\
\hline$\geq 50$ & 28 & $4(14.3)$ & 0.26 & 2.14 & $0.19-26.00$ & 0.260 \\
\hline$<50$ & 34 & $2(8.3)$ & & & & \\
\hline \multicolumn{7}{|l|}{ Sex } \\
\hline Male & 38 & $6(15.8)$ & 0.01 & - & - & 0.009 \\
\hline Female & 24 & 0 & & & & \\
\hline \multicolumn{7}{|l|}{ Height (cm) } \\
\hline$\geq 165$ & 31 & $4(12.9)$ & 0.39 & 4.86 & $0.27-159.00$ & 0.280 \\
\hline$<165$ & 31 & $2(6.5)$ & & & & \\
\hline \multicolumn{7}{|c|}{ Body mass index $\left(\mathrm{kg} / \mathrm{m}^{2}\right)$} \\
\hline$\geq 21$ & 31 & $2(6.5)$ & 0.39 & 4.38 & $0.50-98.00$ & 0.190 \\
\hline$<21$ & 31 & $4(12.9)$ & & & & \\
\hline \multicolumn{7}{|l|}{ Approach } \\
\hline Laparoscopy & 37 & $6(16.2)$ & 0.01 & - & - & 0.022 \\
\hline Open & 25 & 0 & & & & \\
\hline \multicolumn{7}{|l|}{ Operative indication } \\
\hline Cancer/dysplasia & 49 & $5(10.2)$ & 0.78 & 1.63 & $0.02-110.00$ & 0.810 \\
\hline Inflammation & 13 & $1(7.7)$ & & & & \\
\hline
\end{tabular}

$\mathrm{OR}$, odds ratio; $\mathrm{Cl}$, confidence interval.

Table 3. Comparison of Measured Values in Patients Who Were Scheduled to Undergo Laparoscopic RPC with Handsewn Anastomosis

\begin{tabular}{lccc}
\hline & \multicolumn{2}{c}{$\begin{array}{c}\text { Conversion to stapled } \\
\text { anastomosis }\end{array}$} & P-value \\
\cline { 2 - 3 } & No $(\mathrm{n}=31)$ & Yes $(\mathrm{n}=6)$ & \\
\hline tSMA to AC/rSMA to AC & $0.309 \pm 0.017$ & $0.379 \pm 0.038$ & 0.096 \\
tSMA to AC $(\mathrm{mm})$ & $95.0 \pm 5.4$ & $116.0 \pm 12.0$ & 0.130 \\
d-Sci $(\mathrm{mm})$ & $108.0 \pm 2.0$ & $106.0 \pm 4.5$ & 0.700 \\
d-inlet $(\mathrm{mm})$ & $114.0 \pm 2.3$ & $114.0 \pm 4.9$ & 0.980 \\
d-outlet $(\mathrm{mm})$ & $87.7 \pm 1.4$ & $81.5 \pm 3.1$ & 0.076 \\
\hline
\end{tabular}

Values are presented as mean \pm standard deviation.

RPC, restorative proctocolectomy; SMA, superior mesenteric artery; tSMA, terminal of ileal branch of the SMA; rSMA, root of the SMA; $A C$, upper margin of the anal canal; $d-S c i$, the distance between the ischium at the femoral head; $d$-inlet, the distance between the promontory angle and the supra pubic margin; d-outlet, the distance between the coccyx and the inferior pubic margin.

sion of the SMA branch and fenestration of the mesentery were performed in 28 patients (75.7\%). Male sex remained a risk factor of non-reaching $(P=0.02)$.

When the data were divided into 2 groups based on the median distances, the group having a distance $\geq 11 \mathrm{~cm}$ between the tSMA and the AC $(P=0.045)$ had a greater risk of non-reaching. The small d-outlet group showed a tendency for non-reaching, but the difference did not reach statistical significance.

\section{DISCUSSION}

Our study findings suggest that laparoscopic RPC with handsewn anastomosis may limit the extension of the ileal pouch and its induction into the anus and that measuring the distance from the terminal SMA to the anus by preoperative CT may predict the tendency for non-reaching.

RPC with handsewn anastomosis with mucosectomy is a difficult procedure for general surgeons; thus, stapled anastomosis without mucosectomy has become widely implemented. However, it has been suggested that mucosectomy offers several important advantages, because leaving the diseased mucosa exposes the patient to the risk of carcinogenesis from 
Table 4. Risk Factors for Conversion from Handsewn to Stapled Anastomosis in Laparoscopic IPAA

\begin{tabular}{|c|c|c|c|}
\hline Risk factor & No. & $\begin{array}{c}\text { Conversion } \\
\text { to stapled } \\
\text { anastomosis, } \\
\text { No. (\%) }\end{array}$ & $P$-value \\
\hline Total & 37 & $6(16.2)$ & \\
\hline Age (yr) & & & 0.330 \\
\hline$\geq 50$ & 18 & $4(22.2)$ & \\
\hline$<50$ & 19 & $2(10.5)$ & \\
\hline Sex & & & 0.020 \\
\hline Male & 25 & $6(24.0)$ & \\
\hline Female & 12 & 0 & \\
\hline Height (cm) & & & 0.390 \\
\hline$\geq 165$ & 23 & $4(17.4)$ & \\
\hline$<165$ & 14 & $2(14.3)$ & \\
\hline Body mass index $\left(\mathrm{kg} / \mathrm{m}^{2}\right)$ & & & 0.490 \\
\hline$\geq 21$ & 17 & $2(11.8)$ & \\
\hline$<21$ & 20 & $4(20.0)$ & \\
\hline Operative indication & & & 0.970 \\
\hline Neoplasm & 31 & $5(16.1)$ & \\
\hline Inflammation & 6 & $1(16.7)$ & \\
\hline HALS & & & 0.220 \\
\hline Yes & 4 & 0 & \\
\hline No & 33 & $6(18.2)$ & \\
\hline tSMA to $\mathrm{AC} / \mathrm{rSMA}$ to $\mathrm{AC}$ & & & 0.063 \\
\hline$\geq 0.672$ & 18 & $5(27.8)$ & \\
\hline$<0.672$ & 18 & $1(5.6)$ & \\
\hline tSMA to $\mathrm{AC}(\mathrm{mm})$ & & & 0.045 \\
\hline$\geq 110$ & 17 & $5(29.4)$ & \\
\hline$<110$ & 19 & $1(5.3)$ & \\
\hline d-Sci (mm) & & & 0.410 \\
\hline$\geq 107$ & 18 & $2(11.1)$ & \\
\hline$<107$ & 19 & $4(21.1)$ & \\
\hline $\mathrm{d}$-inlet $(\mathrm{mm})$ & & & 1.000 \\
\hline$\geq 115$ & 17 & $3(17.6)$ & \\
\hline$<115$ & 17 & $3(17.6)$ & \\
\hline $\mathrm{d}$-outlet $(\mathrm{mm})$ & & & 0.090 \\
\hline$\geq 88$ & 16 & $1(16.7)$ & \\
\hline$<88$ & 18 & $5(62.5)$ & \\
\hline
\end{tabular}

IPAA, ileal pouch-anal anastomosis; HALS, hand-assisted laparoscopic surgery; SMA, superior mesenteric artery; tSMA, terminal of ileal branch of the SMA; rSMA, root of the SMA; $A C$, upper margin of the anal canal; $\mathrm{d}$-Sci, the distance between the ischium at the level of the femoral head; $d$-inlet, the distance between the promontory angle and the suprapubic margin; $d$-outlet, the distance between the coccyx and the inferior pubic margin. the residual mucosa. ${ }^{4}$

Various techniques for lengthening the mesentery have been studied, but there are cases in which RPC with handsewn anastomosis is technically difficult. Of the 1,789 IPAAs performed in the Mayo Clinic, IPAA could not be performed in 32 (1.8\%) for technical reasons such as severe obesity, mesenteric obesity, and ischemia of the pouch region of the intestinal tract due to mesenteric distraction. A multivariate analysis reported that only age $>40$ years was a risk factor in that cohort. ${ }^{5}$

The frequency of high obesity and mesenteric obesity is reportedly lower in Japan than in Western countries. Nevertheless, there are a certain number of cases in whom handsewn anastomosis is not possible. Ikeuchi et al. ${ }^{23}$ reported that RPC with handsewn anastomosis was possible in 923 (97.8\%) of 944 RPCs, at a center with a high volume of RPC surgery. Although such centers may be familiar with the procedure, the possibility of the cases reflecting open surgery must be considered.

The laparoscopic IPAA procedure is more complicated than open surgery. The steps include mobilization and vascular division of the entire colon, dissection around the rectum to immediately above the anal canal, dissection of the terminal ileum, confirmation of ileal reaching, mesenteric lengthening, including vascular division and fenestration of the mesentery, additional mobilization, pouching preparation, mucosectomy from the anus, specimen removal, guiding the pouch to the anus, and performing a handsewn anastomosis. In these processes, it is often necessary to repeat the laparoscopic operation and create an alternative small laparotomy. In addition, it is difficult to reach the anus laparoscopically and to pull the pouch down sufficiently. Consequently, we considered it to be plausible that laparoscopic surgery was a risk factor for nonreaching. Consequently, in cases where mucosectomy is essential due to medical conditions (in cases where there is dysplasia of the rectum, cancer, or severe inflammation of the lower rectum), but where it is difficult for the pouch to reach the anus under laparoscopic conditions, conversion to laparotomy should be considered. In our series, handsewn anastomosis was possible in all the 6 cases of hand-assisted laparoscopic surgery in addition to all the 25 cases of open surgery, which may indicate keeping pushing the pouch into the pelvis by hand until transanal anastomosis is completed is very useful for making the pouch reach. We think we should not hesitate to convert to open surgery in cases the complete rectal mucosectomy is essential.

Given the difficulty of laparoscopic IPAAs, it is considered 
important to identify factors that predict non-reaching preoperatively. Ohira et al. ${ }^{17}$ reported that mesenteric length, predictable from a CT scan, was a significant risk factor. They used terminal levels of ICA (tICA) that could be followed by CT and concluded that reaching of the pouch was problematic when the distance between the IICA and the AV exceeded $21 \mathrm{~cm}$. We suspected that the tSMA was closer to the apex of the pouch and was a more sensitive indicator, and that the $\mathrm{AC}$ was more accurate than the AV. We showed that reaching of the pouch was difficult when the distance between the tSMA and AC exceeded $11 \mathrm{~cm}$. Our results, similar to the Ohira et al. ${ }^{17}$ study, showed that the pouch could more easily reach the anus in cases where the tSMA was more caudal. tICA to AV longer than $21 \mathrm{~cm}$ and tSMA to AC longer than $11 \mathrm{~cm}$ seems a considerable difference, but Ohira, et al. used the AV as a landmark while we used the upper edge of the anal canal. We thought tSMA might be more adaptable landmark compared to tICA because position of the apex of the J-pouch is more important than that of the ileocecum. On a CT scan, the small intestine falls into the small pelvis in some cases, but not in others. IPAA may be easier to perform in the former type of cases.

Furthermore, in our experience, performing surgery on cases with a narrow pelvis or with obesity is even more difficult. We investigated whether these anatomical factors could be risk factors. The indicator on pelvic size was based on a study by Akiyoshi et al. ${ }^{24}$ who evaluated the relationship between pelvic size and difficulty of laparoscopic surgery for rectal cancer. No previous studies had attempted to analyze pelvic size as a factor in IPAA. Although no significant difference was found in this study, it was suggested that the d-outlet may be a useful index. The last step of retracting the pouch laparoscopically into the anus requires much skill, and the results are consistent with clinical experience. Strictly, we could not know if the pouch really would not reach the anus without trying. However, predicting "reachability" preoperatively may be quite useful.

The greatest advantage of this study is that none of the CT indices used required complicated calculations or three-dimensional reconstructions and could be easily obtained before surgery in any hospital. However, our study had several limitations. First, the findings were based on single-center data and the sample size was small. Because of the nature of this operation, it is difficult to collect a large sample size, and thus there is a need for studies that analyze multicenter data. Second, information of preoperative backwash ileitis was missing. Chronic inflammation could shorten the length of intestine. At least, there was no backwash ileitis in 6 cases who were need- ed to conversion to stapled anastomosis. Third, because no CT scans were available for older cases, no anatomical study was possible with open IAA. However, we could analyze anatomical features of all laparoscopic IAAs, which may pose a high risk of non-reaching. It is also important to seek further indicators in future. Fourth, tSMA could be modality and imaging condition dependent.

In conclusion, it may be more difficult to extend the pouch up to the anus in laparoscopic IPAA than in laparotomy. In particular, for men and individuals whose preoperative distance from the tSMA to the AC on preoperative CT images exceeds $11 \mathrm{~cm}$, it is necessary to consider in advance that IPAA with handsewn anastomosis may need to be abandoned, and to prepare to change over to stapled anastomosis or open surgery if necessary.

\section{ADDITIONAL INFORMATION}

\section{Funding Source}

This work was supported by Grants-in-Aid for Scientific Research (C: grant number; 19K09137, 19K09115).

\section{Conflict of Interest}

No potential conflict of interest relevant to this article was reported.

\section{Data Availability Statement \\ Not applicable.}

\section{Author Contribution}

Conceptualization: Emoto S. Data curation: Emoto S. Formal analysis: Emoto S. Funding acquisition: Emoto S, Tanaka T, Ishihara S. Investigation: Emoto S. Methodology: Emoto S, Nishikawa T, Shuno Y. Project administration: Emoto S. Resources: Emoto S, Iida Y, Ishii H, Yokoyama Y, Anzai H. Software: Emoto S. Supervision: Hata K. Validation: Emoto S. Visualization: Emoto S. Writing - original draft: Emoto S. Writing - review \& editing: Nozawa H, Kawai K, Sasaki K, Kaneko M, Murono K, Sonoda H, Ishihara S. Approval of final manuscript: all authors.

\section{ORCID}

Emoto S https://orcid.org/0000-0002-6146-9385

Hata K https://orcid.org/0000-0003-4064-8701

Nozawa H https://orcid.org/0000-0002-7891-5986

Kawai K https://orcid.org/0000-0002-5881-0036 
Tanaka T

Nishikawa T

Sasaki K

Kaneko M

Murono K

Iida Y

Ishii H

Anzai H

Ishihara S

\section{REFERENCES}

1. Parks AG, Nicholls RJ. Proctocolectomy without ileostomy for ulcerative colitis. Br Med J 1978;2:85-88.

2. Heald RJ, Allen DR. Stapled ileo-anal anastomosis: a technique to avoid mucosal proctectomy in the ileal pouch operation. $\mathrm{Br}$ J Surg 1986;73:571-572.

3. Derikx LA, Kievit W, Drenth JP, et al. Prior colorectal neoplasia is associated with increased risk of ileoanal pouch neoplasia in patients with inflammatory bowel disease. Gastroenterology 2014;146:119-128.

4. Nobel T, Khaitov S, Greenstein AJ. Controversies in J pouch surgery for ulcerative colitis: a focus on handsewn versus stapled anastomosis. Inflamm Bowel Dis 2016;22:2302-2309.

5. Browning SM, Nivatvongs S. Intraoperative abandonment of ileal pouch to anal anastomosis: the Mayo Clinic experience. J Am Coll Surg 1998;186:441-445.

6. Smith L, Friend WG, Medwell SJ. The superior mesenteric artery: the critical factor in the pouch pull-through procedure. Dis Colon Rectum 1984;27:741-744.

7. Cherqui D, Valleur P, Perniceni T, Hautefeuille P. Inferior reach of ileal reservoir in ileoanal anastomosis: experimental anatomic and angiographic study. Dis Colon Rectum 1987;30:365371.

8. Burnstein MJ, Schoetz DJ Jr, Coller JA, Veidenheimer MC. Technique of mesenteric lengthening in ileal reservoir-anal anastomosis. Dis Colon Rectum 1987;30:863-866.

9. Thirlby RC. Optimizing results and techniques of mesenteric lengthening in ileal pouch-anal anastomosis. Am J Surg 1995; 169:499-502.

10. Goes RN, Nguyen P, Huang D, Beart RW Jr. Lengthening of the mesentery using the marginal vascular arcade of the right colon as the blood supply to the ileal pouch. Dis Colon Rectum 1995;38:893-895.

11. Martel P, Majery N, Savigny B, Sezeur A, Gallot D, Malafosse M. Mesenteric lengthening in ileoanal pouch anastomosis for ulcerative colitis: is high division of the superior mesenteric pedicle a safe procedure? Dis Colon Rectum 1998;41:862-866.

12. Martel P, Blanc P, Bothereau H, Malafosse M, Gallot D. Comparative anatomical study of division of the ileocolic pedicle or the superior mesenteric pedicle for mesenteric lengthening. Br J Surg 2002;89:775-778.

13. Araki T, Parc Y, Lefevre J, Dehni N, Tiret E, Parc R. The effect on morbidity of mesentery lengthening techniques and the use of a covering stoma after ileoanal pouch surgery. Dis Colon Rectum 2006;49:621-628.

14. Baig MK, Weiss EG, Nogueras JJ, Wexner SD. Lengthening of small bowel mesentery: stepladder incision technique. Am J Surg 2006;191:715-717.

15. Uraiqat AA, Byrne CM, Phillips RK. Gaining length in ileal-anal pouch reconstruction: a review. Colorectal Dis 2007;9:657-661.

16. İsmail E, Açar Hİ, Arslan MN, et al. Comparison of mesenteric lengthening techniques in IPAA: an anatomic and angiographic study on fresh cadavers. Dis Colon Rectum 2018;61:979-987.

17. Ohira G, Miyauchi H, Narushima K, et al. Predicting difficulty in extending the ileal pouch to the anus in restorative proctocolectomy: investigation of a simple predictive method using computed tomography. Colorectal Dis 2017;19:O34-O38.

18. Polle SW, van Berge Henegouwen MI, Slors JF, Cuesta MA, Gouma DJ, Bemelman WA. Total laparoscopic restorative proctocolectomy: are there advantages compared with the open and hand-assisted approaches? Dis Colon Rectum 2008;51: 541-548.

19. Hemandas AK, Jenkins JT. Laparoscopic pouch surgery in ulcerative colitis. Ann Gastroenterol 2012;25:309-316.

20. Hata K, Kazama S, Nozawa H, et al. Laparoscopic surgery for ulcerative colitis: a review of the literature. Surg Today 2015; 45:933-938.

21. Baek SJ, Lightner AL, Boostrom SY, et al. Functional outcomes following laparoscopic ileal pouch-anal anastomosis in patients with chronic ulcerative colitis: long-term follow-up of a case-matched study. J Gastrointest Surg 2017;21:1304-1308.

22. Utsunomiya J, Yamamura T, Kusonoki M, Iwama T. The current technique of ileoanal anastomosis. Dig Surg 1988;5:207214.

23. Ikeuchi H, Uchino M, Matsuoka H, et al. Surgery for ulcerative colitis in 1,000 patients. Int J Colorectal Dis 2010;25:959-965.

24. Akiyoshi T, Kuroyanagi H, Oya M, et al. Factors affecting the difficulty of laparoscopic total mesorectal excision with double stapling technique anastomosis for low rectal cancer. Surgery 2009;146:483-489. 